\title{
On-chip lithium niobate microresonators for photonics applications
}

\author{
Li-Kun Chen ${ }^{1}$, and Yun-Feng Xiao ${ }^{1,2,3,4^{*}}$ \\ ${ }^{1}$ State Key Laboratory for Artificial Microstructure and Mesoscopic Physics, School of Physics, Peking University, Beijing 100871, China; \\ ${ }^{2}$ Nano-optoelectronics Frontier Center of the Ministry of Education (NFC-MOE) \& Collaborative Innovation Center of Quantum Matter, \\ Beijing 100871, China; \\ ${ }^{3}$ Collaborative Innovation Center of Extreme Optics, Taiyuan 030006, China; \\ ${ }^{4}$ Beijing Academy of Quantum Information Sciences, Beijing 100193, China
}

Received July 1, 2019; accepted July 22, 2019; published online October 15, 2019

Citation: $\quad$ L.-K. Chen, and Y.-F. Xiao, On-chip lithium niobate microresonators for photonics applications, Sci. China-Phys. Mech. Astron. 63, 224231 (2020), https://doi.org/10.1007/s1 1433-019-9453-3

Since its first synthesis in 1960, lithium niobate $\left(\mathrm{LiNO}_{3}, \mathrm{LN}\right)$ has been widely used in modern optoelectronics industry and nonlinear optics. Its high second-order susceptibility and wide transmission window render $\mathrm{LN}$ an excellent platform for applications ranging from frequency conversion to phase modulation. Over the past few years, LN microcavities have attracted intense interest for applications in nonlinear photonics. This scheme combines the crystalline merits of LN and the enhanced lightmatter interaction of whispering gallery mode (WGM) microcavities [1-4]. Owing to the commercial implementation of lithium niobate on insulator (LNOI) wafers, on-chip microstructured LN devices, such as optical waveguides and gratings, have been successfully developed. On this basis, novel photonics applications, including efficient electro-optical modulation, optical parametric conversion and optical combs, have been realized at the chip scale [5-7].

A commercially available LNOI wafer (as shown in Figure 1) comprises a thin layer of LN film on top, a bonded layer of silica buffer and a LN substrate layer (NANOLN, Co.). In 2014, the fabrication processes of microstructured LN devices on this commercially available wafer were first demonstrated using femtosecond laser micromachining followed by focused ion beam milling. Subsequently, argon plasma $(\mathrm{Ar}+)$ in a reactive ion etching system was used to etch the LNOI to produce similar devices in batch production

*Corresponding author (email: yfxiao@pku.edu.cn)
[8]. Recently, the former fabrication technique was improved by Lin et al. [9], and the reported $Q$ factor of a single microdisk reached $9 \times 10^{6}$. As shown in Figure 1, the fabrication process is as follows [10]: (i) A tightly focused femtosecond laser beam is used to ablate both the top LN layer and silica buffer bonded layer to form a cylindrical post. (ii) The sidewall of the cylindrical post is smoothed using FIB polishing. (iii) The silica layer is selectively removed by chemical wet etching, resulting in a silica pedestal to support the freestanding LN microdisk. Notably, the $Q$ factor can be further enhanced to $1.5 \times 10^{7}$ by mechanical polishing [11].

Due to its large second-order susceptibility and wide transmission window, LN is naturally a superior choice for frequency conversion applications, such as second harmonic generation and difference frequency generation. To achieve highly efficient frequency conversion, the phase matching condition of nonlinear processes must be satisfied. Typically, the perfect phase matching is achieved owing to the birefringence of LN, which is difficult to achieve simultaneously for two double resonances in a cavity. Although quasi phase matching conditions can be achieved by fabricating a periodically poled LN (PPLN) structure, such LN structure considerably spoil the $Q$ factors of the cavity due to scattering losses [12]. Different from these techniques, Cheng and collaborators [9] reached effective quasi phase matching condition in an X-cut LN microdisk to obtain an extremely high conversion efficiency. In their scheme, both the refractive 


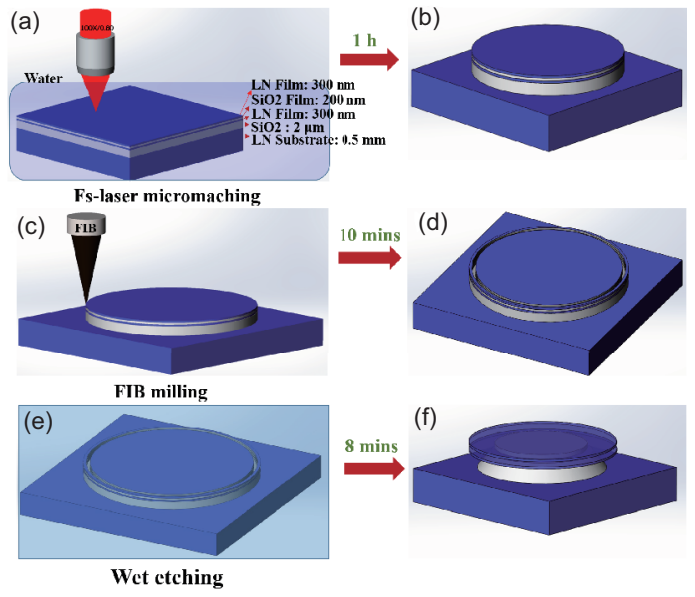

Figure 1 (Color online) Fabrication process of LN microdisks. (a), (b) Writing is applied for a LN cylinder using focused femtosecond laser beam micromachining; (c), (d) the sidewall is polished using FIB milling; (e), (f) the silica layer is removed by HF wet etching.

indices of fundamental/harmonic modes and nonlinear coefficients vary along the periphery of microdisk. The mismatching of fundamental and harmonic wave vectors can be compensated by an effective nonlinear coefficient. Compared with the traditional quasi phase matching condition constructed in a PPLN structure, the mismatching oscillates within a certain range, allowing multiple choices of compensation, thereby exhibiting the potential for broadband phase matching [13]. The second harmonic conversion efficiency achieves up to $9.9 \% / \mathrm{mW}$, which is at least two orders of magnitude higher than the values reported in former works in LN microdisks [14-17].

Because of its relatively high refractive index, $\mathrm{LN}$ is also a superior platform for studying slot structures, which support exterior optical modes and have great potential in precision sensing and surface nonlinearity [18]. Thus far, slot structures have been fully realized in silica/silicon waveguides and surface plasmonic structures [19], of which the fineness is inevitably limited by either surface roughness or metallic intrinsic losses. Cheng and collaborators [20] reported the fabrication of a double layer LNOI microdisk with a gap of $128 \mathrm{~nm}$. In contrast to a wafer with a single LN layer, the double-layer LN film was specially designed to fabricate a double disk cavity with a slot structure. The $Q$ factor of the slot WGM reaches $1.2 \times 10^{5}$, where scattering and absorption losses can be suppressed by applying a crystalline nanofilm with subnanometer surface roughness [20]. The double disk microstructure features strong optical nonlinearity and optomechanical compatibility, which provide a simple platform for studying passive synchronization and cooling.

The novel techniques developed by Y. Cheng, X. Chen and collaborators for the fabrication of LNOI microstructures present a promising future for nonlinear integrated devices. This controllable and reproducible fabrication process based on femotosecond laser ablation can considerably promote fundamental LN-related research, including the development of optical parametric conversion and optomechanics.

This work was supported by the National Key R\&D Program of China (Grant No. 2016YFA0301302), and the National Natural Science Foundation of China (Grant Nos. 11825402, 11654003, 61435001, and 11527901). The authors thank S.-J. Tang and Y. Zhi for their helpful discussions.

1 K. J. Vahala, Nature 424, 839 (2003).

2 Q. H. Song, Sci. China-Phys. Mech. Astron. 62, 074231 (2019)

3 G. Y. Chen, J. F. Liu, Y. C. Yu, R. M. Liu, G. X. Zhu, Y. Z. Chen, Z. X. Chen, and X. H. Wang, Sci. China-Phys. Mech. Astron. 62, 064211 (2019).

4 L. H. Chen, G. Y. Chen, R. M. Liu, and X. H. Wang, Sci. China-Phys. Mech. Astron. 62, 974211 (2019).

5 Y. Li, C. Wang, and M. Loncar, Opt. Lett. 40, 2902 (2015).

6 C. Wang, M. Zhang, X. Chen, M. Bertrand, A. Shams-Ansari, S. Chandrasekhar, P. Winzer, and M. Lončar, Nature 562, 101 (2018).

7 C. Wang, M. Zhang, M. Yu, R. Zhu, H. Hu, and M. Loncar, Nat. Commun. 10, 978 (2019), arXiv: 1809.08637.

8 M. Zhang, C. Wang, R. Cheng, A. Shams-Ansari, and M. Lončar, Optica 4, 1536 (2017).

9 J. Lin, N. Yao, Z. Hao, J. Zhang, W. Mao, M. Wang, W. Chu, R. Wu, Z. Fang, L. Qiao, W. Fang, F. Bo, and Y. Cheng, Phys. Rev. Lett. 122, 173903 (2019).

10 J. Lin, Y. Xu, Z. Fang, M. Wang, J. Song, N. Wang, L. Qiao, W. Fang, and Y. Cheng, Sci. Rep. 5, 8072 (2015).

11 R. Wu, M. Wang, J. Xu, J. Qi, W. Chu, Z. Fang, J. Zhang, J. Zhou, L. Qiao, Z. Chai, J. Lin, and Y. Cheng, Nanomaterials 8, 910 (2018).

12 Z. Z. Hao, L. Zhang, A. Gao, W. B. Mao, X. D. Lyu, X. M. Gao, F. Bo, F. Gao, G. Q. Zhang, and J. J. Xu, Sci. China-Phys. Mech. Astron. 61, 114211 (2018).

13 X. Jiang, L. Shao, S. X. Zhang, X. Yi, J. Wiersig, L. Wang, Q. Gong, M. Lončar, L. Yang, and Y. F. Xiao, Science 358, 344 (2017).

14 J. Lin, Y. Xu, J. Ni, M. Wang, Z. Fang, L. Qiao, W. Fang, and Y. Cheng, Phys. Rev. Appl. 6, 014002 (2016).

15 L. Wang, C. Wang, J. Wang, F. Bo, M. Zhang, Q. Gong, M. Lončar, and Y. F. Xiao, Opt. Lett. 43, 2917 (2018).

16 R. Luo, H. Jiang, S. Rogers, H. Liang, Y. He, and Q. Lin, Opt. Express 25, 24531 (2017).

17 J. T. Lin, Y. X. Xu, Z. W. Fang, M. Wang, N. W. Wang, L. L. Qiao, W. Fang, and Y. Cheng, Sci. China-Phys. Mech. Astron. 58, 114209 (2015), arXiv: 1405.6473.

18 X. Zhang, Q. T. Cao, Z. Wang, Y. Liu, C. W. Qiu, L. Yang, Q. Gong, and Y. F. Xiao, Nat. Photon. 13, 21 (2019).

19 Y. F. Xiao, C. L. Zou, B. B. Li, Y. Li, C. H. Dong, Z. F. Han, and Q. Gong, Phys. Rev. Lett. 105, 153902 (2010), arXiv: 1011.0251.

20 Y. Zheng, Z. Fang, S. Liu, Y. Cheng, and X. Chen, Phys. Rev. Lett. 122, 253902 (2019). 